\title{
"Moving Forward": Older Adult Motivations for Group-Based Physical Activity After Cancer Treatment
}

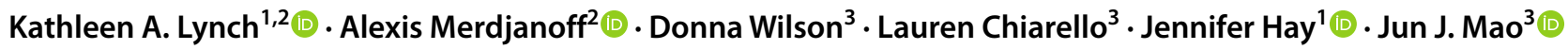

Accepted: 7 August 2021 / Published online: 28 September 2021

(c) International Society of Behavioral Medicine 2021

\begin{abstract}
Background Engagement in physical activity (PA) post-treatment can improve health outcomes and quality of life among cancer survivors. The purpose of this study is to explore United States (US) older adult cancer survivors' (OACS) reasons for engaging in group-based PA classes, to identify themes supporting exercise motivations in the context of cancer recovery.

Methods OACS participating in a fitness program at a large US comprehensive cancer center completed semi-structured interviews. Transcripts were analyzed using modified grounded theory, and demographic data were analyzed descriptively. Results Modified grounded theory analysis ( $n=25$; age $M=70.92, \mathrm{SD}=10.82 ; 9$ cancer types) identified individual rationales for exercise grounded in collective experience. Participants' internal motivations for PA are shaped by the desire for control over an uncertain future and post-treatment body, obtained by literally "moving forward" post-cancer; this is supported by external motivations for social connections that present a positive model of survivorship, within a setting that instills confidence and safety.

Conclusions Exercise can be a way for older adults to tap into internal and external motivations that support cancer survivorship. Interventions that make explicit connections between exercise and cancer recovery, facilitate interpersonal interaction, and promote a sense of safety may be the most effective. The concepts identified in this study can inform the development of future interventions to improve long-term behavior change among OACS and evaluate existing PA programs.
\end{abstract}

Keywords Qualitative · Older adult · Physical activity · Cancer · Survivorship · Motivation

\section{Introduction}

As the United States (US) population ages, the number of older adult cancer survivors is anticipated to increase by approximately 9.4 million over the next two decades [1]. Currently, $64 \%$ of cancer survivors are aged 65 or older; by 2040 , it is estimated to be $73 \%$ [1]. Recent data also

Kathleen A. Lynch

kal587@nyu.edu

1 Department of Psychiatry and Behavioral Science, Memorial Sloan Kettering Cancer Center, 641 Lexington Avenue, 7th Floor, New York, NY 10022, USA

2 Department of Social and Behavioral Sciences, School of Global Public Health, New York University, 715 Broadway, New York, NY 10003, USA

3 Department of Integrative Medicine, Memorial Sloan Kettering Cancer Center, 1429 1st Avenue, New York, NY 10021, USA indicates that the probability of developing cancer doubles for all tumor sites starting at age 60 [2]. Given this projected demographic shift, it is in the interest of public health to increase emphasis on research and interventions to support cancer survivorship among older adults. Recent studies have indicated that involvement in exercise-either through general physical activity (PA) or a structured fitness regimen-has positive impacts on both physical and psychosocial quality of life among cancer survivors and can reduce risk of cancer recurrence [3-9]. However, OACS are often excluded from PA research [10], leading to an evidence gap which may influence the way physicians and patients frame the relevance and value of exercise in the context of cancer survivorship [11].

There has been a lack of inquiry into the rationale, or potential motivating factors, that contribute to voluntary exercise among this population. This gap is significant not only due to the projected US epidemiology of cancer survivorship over the next two decades, but OACS may also 
have unique needs and psychosocial motivations that current PA programming does not address. For example, approximately $25 \%$ percent of OACS have five or more comorbid conditions, which may impact PA engagement and comfort [12]. A recent meta-analyses of PA interventions for cancer survivors also found that interventions involving older populations were less likely to be effective at facilitating maintenance of the behavior post-study [13]. Understanding OACS personal rationale for PA participation may be key developing meaningful exercise interventions that support psychosocial quality of life during cancer recovery.

\section{PA Motivations in the Context of Cancer Survivorship}

Previous research has indicated that exercise participation rates decline in cancer survivors who have undergone treatment; one study found that exercise was the least likely positive behavior change to occur after cancer diagnosis and treatment [14]. A systematic review found that less than $30 \%$ of cancer survivors meet recommended PA guidelines [15]. Behavioral scientists have suggested that clinicians can influence intentions to exercise by targeting known motives for exercise among a particular survivor group [16]. However, while previous studies have evaluated the implementation of PA programs for older adults, there have been no studies which specifically explore OACS motivations for fitness behaviors $[17,18]$.

In non-oncologic contexts, synthesis of recent qualitative studies provides preliminary insight into older adult PA motivation. These motivations tend to include both internal (i.e., personal, psychological) and external (i.e., contextual, socioenvironmental) elements. For instance, a recent metaanalysis of older adult PA in non-clinical contexts identified social interaction (external) and short-term functional outcomes (internal) as primary motivators for participating in exercise interventions [19]. Similarly, a systematic review and qualitative meta-ethnography from the UK theorized that PA contributes to self-esteem among older adults by fostering feelings of purpose (internal), a sense of belonging in collective group activity, and facilitating routine and structure (external) [20].

However, the unique experiences and stressors associated with cancer survivorship may complicate the previously documented exercise motivations of older adults in the general population. Unlike other aspects of the cancer journey which are marked by definitive endpoints-i.e., completing a surgery, the last chemotherapy session-cancer "survivorship" extends throughout the life course [21]. What it means to be a "cancer survivor" is largely self-defined, yet the Institute of Medicine outlines the "survivorship" period as spanning from the time of diagnosis to the end of life, or until cancer recurrence [22]. This caveat, "until cancer recurrence," places an asterisk on survivorship for many cancer survivors, contributing to notions of being "cancer-free but not free of cancer" [21]. Fears of recurrence, the sense of being "tethered" to cancer, have been linked to anxiety among cancer survivors [23, 24].

Older cancer patients have a complex relationship with the concept of survivorship [25], and may experience distinct motivations for PA. A grounded theory study among patients over the age of 70 who completed treatment for breast cancer found that survivorship was conceptualized as a tenuous, impermanent state [25]. Previous study suggests that OACS draw on internal and external sources of strength to cope with cancer recovery and survivorship maintenance [26], and qualitative study among older breast cancer survivors found that older adults tend to draw on an intrinsic sense of self-reliance and optimism, built through years of lived experience. By drawing continuity from their "previous" to their "current" self, one could "move on with life" despite the uncertainty of a cancer-free future [25, 26]. Yet, the study also found that this self-reliance is embedded in social connection [26]. Considering this finding in light of the collective sense of belonging experienced by healthy older adults in the studies above, group exercise may be a conduit to tap into these sources of internal strength during survivorship.

\section{Evaluating OACS Fitness Classes: Gaps and Limitations}

There is a clear gap in the literature on PA rationale among OACS who are currently, voluntarily active in fitness classes outside the context of a clinical trial. Previous qualitative studies of older adults and exercise, including those from the USA, Denmark, and Australia, either involve individuals who are not currently active, not cancer survivors, or elicit feedback tied to a specific intervention or trial [17, 27-32].

A recent survey of breast cancer survivors in the USA found that over $50 \%$ respondents had an interest in structured exercises classes at a cancer center [33]. A recent systematic analysis found that $56 \%(n=25)$ of National Cancer Institutedesigned Comprehensive Cancer Centers in the USA currently offer fitness classes for their cancer patients [34]. In the community setting, the YMCA Livestrong program is a no-cost PA program for any cancer survivor over the age of 18 , with classes that focus on strength, flexibility, balance, and social support [35]. While barriers to participation in these programs have been explored [36], to date, there have been no studies of what motivates OACS to participate in group-based programs.

Understanding the specific reasons why OACS may voluntarily engage in PA in a specific context, such as a hospital or community-based setting, can help inform the development of sustainable interventions that take place in 
that context. The purpose of the present study is to examine a currently well-attended OACS fitness program, to understand OACS self-identified rationale for attending. By identifying the components of the program that keep participants engaged, from the perspective of the participants themselves, we can uncover potential themes to support longterm behavior change and inform effective PA programming for this population.

\section{Methods}

\section{Data Source}

Data for this study were collected as part of an ongoing program evaluation of the Integrative Medicine Service (IMS) at a large US comprehensive cancer center, which used ethnographic observations and semi-structured interviews to explore patient perspectives and experiences across IMS program offerings. For the present analysis, a retrospective research protocol was reviewed and approved by the center's Institutional Review Board.

\section{Participants and Procedures}

Individuals were eligible to participate in the study if they had consented, as part of their usual care, to take part in an IMS therapy. For the present analysis, participants also needed to be an OACS who had attended at least one IMS fitness class. In this study, we defined OACS as a patient over the age of 60 with a history of cancer. To obtain perspectives from patients with diverse cancer histories, there were no restrictions on time since diagnosis or treatment status. Participants were recruited from three IMS fitness classes: Focus Fitness for Breast Cancer Survivors (which focuses on strengthening pectoral muscles and facilitating lymphatic flow; populated by late-middle age or older female patients), Exercise for All (which includes seated exercises for individuals with restricted mobility; populated by older male and female patients of diverse cancer types), and Strong Bones (which is for individuals with osteoporosis or osteopenia; populated by largely older female patients of diverse cancer types). After explaining the study at the end of each fitness class, a researcher who acted as a participant observer during the class (KAL) approached class attendees and asked if they would be willing to participate in a semistructured interview. After recruiting 10 participants in an initial convenience sample, the remaining individuals were purposively sampled based on age, gender, and cancer survivorship. We also sampled one cancer survivor from each class (Focus Fitness, Exercise for All, Strong Bones) under the age of 60 who attended at least 1 class per week over the 16-week data collection period. These interviews served as "key informant" interviews supplement the researchers' observational fieldnotes about the structure and social setting of each class, and to facilitate further recruitment. Participants were enrolled to thematic saturation, which was defined in this study as the point when all relevant themes were fully explored and no new themes emerged from at least two subsequent interviews [37]. Saturation was reached by 25 interviews, consistent with previous literature on qualitative sample sizes for single-site exploratory studies [37].

Interviews occurred in person between July 8 and September 10, 2019, and were moderated by a researcher experienced in qualitative methods (KAL). At the start of each session, the researcher described the project, gave information about what study participation would entail, and steps to maintain confidentiality. The voluntary nature of the study was emphasized. Participants were asked for their consent to audio record the interview. Interviews lasted between 20-50 min.

\section{Interview Structure}

At the start of the session, participants completed a brief questionnaire assessing age, gender, ethnicity, cancer diagnosis, and self-rated health and well-being using items from the PROMIS Scale v. 1.2: Global Health. This is a validated measure consisting of ten questions on a 5-point Likert scale asking participants to self-rate various dimensions of physical and psychological health over the past 7 days [38]. Interviews followed a semi-structured interview guide, covering the following topics: how individuals came to participate in the fitness program, previous and current exercise habits, motivations and barriers, and recommendations for program improvement (Appendix I). The interview guide also included subsidiary probing questions to enable participants to elaborate on specific aspects of their experience [39].

\section{Analysis}

Items from the demographic questionnaire were de-identified and analyzed descriptively in Stata 15.1 [40]. Frequencies were calculated for categorical data: means, medians, and standard deviation; the range was calculated for all continuous data. The PROMIS measure was scored according to a validated scoring manual [38]. Interviews were audiorecorded using an encrypted device, de-identified, and transcribed verbatim. Transcripts were analyzed by two experienced qualitative researchers (KAL, AM) according to an iterative process of modified grounded theory [41, 42]. This modified approach, popularized by Charmaz [41, 42] who posits that "the analysis, which relates to time, culture, and context, reflects both the participant's and researchers' way of thinking [...] the story reflects the viewer as well as the viewed" [42]. Given that pre-existing behavioral 
frameworks will invariably influence researcher's thinking throughout the analysis, the authors consciously incorporated elements of these frameworks into initial annotation and coding, co-producing meaning from pre-existing literature and the novel, personal narratives of this understudied population. This mode of grounded theory therefore coconstructs knowledge and focuses on meaning and process within a particular social context. Given that the objective was to understand the rationale for exercise among a unique population, this approach was deemed appropriate for the present analysis. A detailed analysis protocol is described in Appendix 2. Qualitative software NVivo Pro 12.0 facilitated the analysis, as well as stored the transcripts and final codebook [43].

\section{Results}

\section{Demographics}

A total of 25 OACS participated in interviews $(M=70.92$, $\mathrm{SD}=10.82)$. The majority were female $(88 \%)$ and white (96\%). Nine malignancy types were reported, the most common being breast (36\%). The mean self-rated mental health score was $52.58(\mathrm{SD}=3.88)$, and mean physical health score was $51.46(\mathrm{SD}=4.62)$. Full participant demographics are displayed in Table 1.

\section{OACS Motivating Factors for Group-Based PA}

Modified grounded theory analysis revealed multi-faceted reasons for participating in group-based PA. In this section, we will explore a continuum of motivation, wherein core internal motivations exist in the context of externally driven, collective experience. As Table 2 illustrates, participant motivations for PA participation are (1) grounded in notions of coping with a precarious future (internal motivation), (2) shift to "moving" forward post-cancer by taking control over the present (internal motivation), (3) are contextualized within a sense of resiliency fostered through social relations (external motivation), and (4) take place in a setting which instills confidence in medicalized recovery. Each of these dimensions will be explored in detail below, grounded in the words of the participants, and situated in the context of the fitness classes.

\section{Internal Motivation: PA Is a Way to Cope with an Unknown Future}

During the interviews, participants discussed the numerous ways that cancer disrupted their sense of stability, physical autonomy, and imagining of the future. Although they were no longer in active treatment, the fear of potential recurrence
Table 1 Participant demographics $(n=25)$

\begin{tabular}{ll}
\hline & $N(\%)$ \\
\hline Mean age (SD, range) & $70.92(11.04,44-91)$ \\
Sex & \\
Male & $3(12)$ \\
Female & $22(88)$ \\
Race & \\
White & $24(96)$ \\
Prefer not to answer & $1(4)$ \\
Cancer history (self-report) & \\
Breast & $9(36)$ \\
Melanoma & $3(12)$ \\
Lymphoma & $1(4)$ \\
Uterine & $2(8)$ \\
Prostate & $1(4)$ \\
Lung & $1(4)$ \\
Colon & $1(4)$ \\
Throat & $1(4)$ \\
Thyroid & $1(4)$ \\
None (caregiver, active surveillance, etc.) & $5(20)$ \\
Mean years of class attendance (SD, range) & $4.10(4.12,0.08-15)$ \\
Primary class attendance & \\
Focused fitness & $4(16)$ \\
Strong bones & $10(40)$ \\
Exercise for all & $11(44)$ \\
PROMIS & \\
Mean general (SD) & $3.83(0.87)$ \\
Mean social activity (SD) & $4.17(0.82)$ \\
Mean GPH T-score (SE, 95\% CI) & $51.46(4.62,42.4-60.5)$ \\
Mean GMH T-score (SE, 95\% CI) & $52.58(3.88,45.0-60.2)$ \\
\hline & \\
\hline & \\
\hline
\end{tabular}

$S D$ standard deviation, $S E$ standard error, $G P H$ global physical health, GMH global mental health

was not far from participants' minds. Often, this fear was articulated when describing the experiences of a friend with cancer. While telling her own story, one participant said,

I recently had a friend who died and [it] was really sad. She was also someone I knew from origami who became one of my cancer friends, and she had had cancer and then she was coming up on her five-year checkup and then she was diagnosed with stage 4 . She had tremendous amounts of chemo and this and that. Nothing was really working. And then she told me well, I have to go on hospice now and she was gone like a week later. It's like, what? It was so weird to me and I still can't even believe she's gone.

In this narrative, she related this painful experience to her own cancer journey and experiences with the fitness class, as someone with little previous experience with exercise: 
Table 2 OACS motivations for group-based PA

\begin{tabular}{|c|c|c|}
\hline Rationale for group-based PA & Description & Dimension of motivation \\
\hline PA is a way to cope with an unknown future & $\begin{array}{l}\text { PA allows participants to cope with the fear and anxi- } \\
\text { ety surrounding a potential cancer recurrence, by } \\
\text { allowing them to feel that they are taking an active } \\
\text { role in their recovery }\end{array}$ & Internal motivations (psychological) \\
\hline $\begin{array}{l}\text { Control over the present: "moving forward" } \\
\text { after cancer through movement }\end{array}$ & $\begin{array}{l}\text { PA increases strength and mobility, enhancing } \\
\text { participant confidence and sense of control over a } \\
\text { physical body changed by cancer treatment }\end{array}$ & \\
\hline Social interactions support resilience & $\begin{array}{l}\text { Class camaraderie functions as a support system, set- } \\
\text { ting for information exchange, and a hopeful model } \\
\text { of cancer recovery }\end{array}$ & External motivations (social and structural) \\
\hline $\begin{array}{l}\text { The physical setting/ context facilitates trust } \\
\text { in pushing physical limits }\end{array}$ & $\begin{array}{l}\text { The hospital-based environment and composition of } \\
\text { the class helped participants feel comfortable trying } \\
\text { new exercises and placed PA in the context of other } \\
\text { recovery goals }\end{array}$ & \\
\hline
\end{tabular}

[A]nd yet even in the face of that I still just don't think... I feel like I'm going to be good. I mean I am not a fit person. I am an overweight person who doesn't like to exercise and lives a sedentary lifestyle. And yet it [the fitness class] has really made a big difference in my life. And you know I do kind of feel like it is you know, the thing that's helping to fight the cancer. I really do.

Other participants similarly noted how living through a constant fear of recurrence, shaped by experiences of other survivors, became a stimulus for PA:

I've learned that I have cancer so in order to battle it from coming back, it's what I put in my mouth and the exercise I do with my body. And that's what's going to help me to fight off anything else coming back.[...] because once you've had cancer, you don't want it back. It comes back in so many different forms. I've had friends who've gotten it back and I'm like, I'm going to do whatever I can to fight it.

As the above excerpts illustrate, participants framed PA as a means of taking an active role in navigating the unknown waters of cancer survivorship. For participants, the fitness class became a way to assert control over future uncertainty:

[I]t's an easy, pleasant way to be more healthy. So you're not sitting at home worrying about a recurrence. You know that you're doing something that's data-based, proved to help.

Participants also framed motivations for PA as the desire to feel "in control" over their body post-treatment, which became a tangible way to cope with fear of recurrence. Cancer treatment can cause dramatic physical changes that persist into survivorship. As one participant stated, "my body was falling apart." The motivation to regain a sense of "control" over one's body was spontaneously expressed in multiple interviews. As one participant stated:

It's just very scary having cancer and I think that-it makes me feel like I'm doing my best to keep it away and-if I exercise. It gives me a little bit more control over my body and ability to feel like I'm doing the best I can.

In certain instances, PA also became a way to refocus mental energy, when impacts of treatment made other aspects of life feel so out of control:

$[\mathrm{H}]$ ormone therapy has a known side effect of - like prednisone makes you starving. I actually gained all that weight back on the prednisone [...] And I sort of felt like that that was an area that was sufficiently out of control that I didn't have faith in my ability to handle it, especially because I did not feel emotionally strong. But this [fitness class] seemed doable.

\section{Internal Motivation:"Moving Forward" Through Movement}

Participants often articulated their motivation for PA as a tangible way to feel like they were "taking control" over a future that could be disrupted at any moment: by a new symptom, the results of the next scan, or the consequences of aging. In the midst of this uncertainty, many spoke about the need to "keep moving" forward, invoking both a literal and metaphorical use of the phrase: "I wasn't afraid of things because I've always felt that whatever you have, to keep moving." In this context, "moving" signifies both physical mobility and a forward progression into post-cancer life. The 
notion of "keeping moving" is articulated as a benefit and a rationale for PA. As one older participant stated: "You know, when you're my age, you need to move [...] You know I say [to myself], 'all right, move, and then I do. It just makes me feel better."

In interviews, participants framed PA as a method to "move on" from their cancer experience by increasing their bodily mobility. As one participant described,

[I]t's basically a good class to get yourself back going. You gain some strength that you lost during the procedure. The immobility--you know, people when they're going through chemo and radiation, they're generally not as active as they were prior to. So, it's very important to get back to the activity, instead of just sitting around, getting up and doing things.

In order to "move forward" post-treatment, participants placed emphasis on regaining mobility. Living with a body changed by cancer treatment was a source of distress for many participants, as one individual tearfully described,

It's really, really important to me to get my body back. I mean it's-I don't want to get upset, but it's what's created such an emotional distress to me to have my body totally-I had to learn how to walk again. I almost died, and so, I had to walk for a long time with a cane. And my body is deformed [...] it's hard to me to have spent years of my life building my muscles to see them gone. My whole body, and my stomach muscles are totally gone.

For some participants, a loss of confidence in physical abilities impacted a core part of their identity. This was especially the case among participants who self-identified as being highly physically active prior to cancer treatment. One participant remembered,

I was growing up in Alaska. And you can imagine... this was like near-frontier. We lived away from civilization and you can imagine the bodily confidence being an important factor. You can do anything; you have to be able to do anything.

For this individual, their way of surviving in the world was predicated on confidence in their physical abilities, and cancer had changed that confidence. In a similar vein, a lifelong athlete described the experience of living in a changed body:

I was a junior in college the point at which Title IX took effect [...] Basically I was always involved in sports so it's really strange for me not to have both the physical capability of doing things and not to feel as though I can run to get to the bus or something like that. That kind of stuff, I really feel like 'whose body am I carrying around?' I feel like an entirely different person. Part of me assumes that I'm going to back into sports and part of me I don't know. But this [fitness class] makes me feel more as though there's a possibility of going in that direction.

As the excerpt above illustrates, in "keeping moving" through regular PA, the "unrecognizable" body is rendered recognizable, enabling participants to "move forward" from their cancer experience. In this sense, psychological experiences of survivorship are grounded in the corporeal, as a strengthened physical body becomes a marker of "productive" recovery.

\section{External Motivation: Resilience Through Social Interaction}

Buttressing the internal motivators of control and movement are the social relationships that develop as a result of fitness class participation. Multiple participants noted group "camaraderie"-one participant even referring to it as an "infectious camaraderie"-as a primary reason they looked forward to returning to the class every week:

Part of what attracts people and what I really like is I love the cohort of women who come. It is like a little peer group and we're quite friendly and out of that have come a number of friendships and people care about each other. So, it's a culture that's been created and I think part of the culture is women who have suffered.

By exercising in a group mainly composed of cancer survivors, participants noted that the class functioned as a support system where they could rely on one another's expertise and experience:

Well the other thing that's interesting too as far as the community, it's not just a social feel good, friendly community, but it's also a really important place to exchange information. There are a lot of times that we sit out here, and somebody will say well, do you know who I could see for this? Or, how does this drug affect you?

The collective experience of the fitness class provided a welcome environment for information exchange and physical goal-setting, as many participants noted that conversations in clinic around PA were either minimal or nonexistent:

It's not something that the NP or the PA or the doctor recommend almost ever [...] when the physician saw me after chemo and right after the surgery visit: okay you can go back to the gym now. And I'm like, con- 
sidering I never went to the gym! They didn't even ask me [...] So I was clueless as to even what that meant.

In the absence of formal clinical communication, the social experience of the fitness classes offered a positive model of PA during cancer survivorship. Participants compared the camaraderie of the fitness classes to a "positive" cancer survivor support group:

And so when I'm in [the] class, you know, we're the survivors, we beat cancer... and so I just feel like it's a very positive vibe. Whereas you know in other therapy like [sitting in] circular situations, there is less of that... and obviously people need this, right? But it wasn't what I needed.

Like the excerpt above, multiple participants favorably compared the fitness classes to "traditional" support groups, noting that a talk therapy group "wasn't what [they] needed." This aligns with the motivation to "keep moving": PA and social engagement became a conduit through which they could process their fears and concerns without those fears becoming the focus of the class itself. In fact, many participants noted that it was "inspiring" to be surrounded by other cancer survivors, and this motivated them to persevere with the exercises:

It was very tough for me, very. Oh my God, I was fatigued. I didn't do anything [...] So why did I come back? Because they were very welcoming, and I felt like I belonged. Like everyone had a different history, a different route, but every one of them had suffered and was coming up, came out on the other side.

The composition of the class acts as a key motivation for PA continuation. By exercising alongside other survivors, participants envision that a future where they "come out on the other side" of cancer is possible. By binding their future with others, health milestones are experienced (and weathered) as a group:

You know, like always somebody coming up to their next test, on their next scan, and we keep track of it. And when [another group member] was in the hospital, we went to visit her.

Group concern for an individual's progress is another way that PA participation enables participants to cope with an uncertain future. As one participant shared, this is an "unanticipated" benefit of the classes that keeps them coming back:

And then we get concerned about the other people like when they go for testing. It freaks us out. Did you get the results? What are they? We don't see him, is he okay? It's kind of, I didn't anticipate that. So, [you] share more because you know people are in similar situations to you. You know you don't have to be embarrassed.

While the physical aspect of the class enables participants to feel a sense of control over the present, the social environment offers optimism, and support, for an uncertain future.

\section{External Motivation: The Physical/Clinical Context Facilitates Trust in Pushing Physical Limits}

Participants' internal notions of control, reinforced by group support, are embedded in the external context of a clinically adjacent setting. In addition to facilitating camaraderie, the environment and composition of the class played a key role in facilitating participant comfort with initiating in PA. Many participants, especially those with a limited history of exercise, noted that they had hesitated initiating exercise on their own, feeling either embarrassed about their posttreatment physical ability, or unsure of what activities were safe to engage in:

Like at the beginning [post-treatment], one of the obstacles to exercise is you don't want to go to your gym [...] I'm a guy and I can barely like lift this two-pound weight. Like a gym will just fill me with horror-I wasn't willing to put myself in that position, because [...] I thought it would make me feel sorry for myself, which I already did. And so, the gym options were not good. And the at-home options were not good, because you don't really know what you're doing. And so this [class] like solved that, solved that problem.

Multiple participants commented on the value of a class comprised specifically of OACS, because it allowed them to try new exercises in a safe, non-judgmental environment. This theme was especially prevalent among participants who had engaged in little to no PA prior to cancer treatment:

I tell people all the time it's a really safe kind of space and you can kind of do it at your own pace, and you don't need to feel conscious that you can't do something, and that if you keep coming to class you will be able to do things you aren't able to do in the first class.

For many participants, the placement of the classes on the hospital campus positioned PA within the context of other treatment regimens and goals. Numerous participants noted that they framed the classes as being "part of their treatment," some even likening it to a prescription:

I tried to think of it as being like a prescription. I tried not to think of it like, oh, I'm going to exercise, because if I would have thought about it that way I never would have gone. [Laughter] But because I 
thought of it like a prescription like, oh I have cancer, I need chemotherapy, great. I need radiation, great. I need exercise class, great. I just was thinking of it that way and that really helped to get me here.

The clinical knowledge of the instructors also created a context where participants felt comfortable pushing themselves out of their physical comfort zone. They felt confident in the knowledge that when given a new or difficult exercise, it was for a clinically relevant reason:

I feel that everybody that takes this class is so lucky because she's a nurse. She knows what it's doing to your body. She knows why, she will check our records. You probably noticed in class sometimes she'll say to somebody, you could handle higher weights by now. Or somebody, don't do this exercise because X. So I think everybody that goes to this class should feel very safe that they're certainly not going to hurt themselves and they can only get better.

The creation of an external space where participants felt confident pushing themselves to "do more than they thought they could" enhanced internal sense of control, sustaining PA participation. As one participant described,

I felt like I could actually do it. It was like, oh look, I'm doing it. [Laughter] You know? And I wasn't expecting to be able to do it. I wasn't. And so at the end of the first class I was like, okay, I did it. I could come back.

Multiple participants describe "feeling accomplished" after the classes, increasing their self-confidence. In this sense, the external environment of the class facilitated internal motivations for participation.

\section{Discussion}

With the rapid increase of aging cancer survivors, helping OACS stay active is a critical public health issue. In this modified grounded theory study, we identified key internal and external themes related to motivations for participating in PA classes. As described in Table 2, OACS internal motivations for PA are shaped by the desire for a sense of control over an uncertain future and post-treatment body, obtained by literally "moving forward." These motivations are reinforced and molded by the social camaraderie with other members of the class, and enacted in a clinical, institutional context. Through engaging in regular PA, OACS "keep moving," gaining strength and self-confidence from their changing physical abilities, and from other class members who present an optimistic model of survivorship. In the context of the fitness class, future uncertainty is quite literally "worked out" in a space where participants feel comfortable pushing past their limits, where they can both confide in others and draw strength.

The results of the present study both support and extend findings from recent meta analyses of older adult PA in nonclinical contexts, where social interaction, increased selfesteem, and short-term functional outcomes were identified as primary motivators for participating in exercise interventions $[19,20]$. By examining motivation in the context of cancer survivorship, social support is found to enhance individual self-efficacy. Through participating in the classes, individuals felt a sense of camaraderie and belonging; "moving together" with others facilitated a belief in their ability to "move forward" in cancer recovery. While previous work on PA in mid-to-later life adults has critiqued health messages encouraging older adults to "move more" or "move faster" [44], in the context of cancer survivorship, older adult participants not only framed their own recovery around movement, but around "moving forward" together.

The continuum of internal and external motivation among OACS reveals that the uncertainties and anxieties of cancer diagnosis and survivorship are a deeply personal, individual experience, but long-term experience of cancer recovery is experienced collectively. Through exercise, participants expressed feeling "productive" and "in control"; they marked control over the future through changes in the body; psychological aspects of cancer recovery are grounded in their physical selves. Yet a strong motivation for returning to the class was experiencing cancer survivorship alongside others; their experiences of recovery are both recognizable and made legible through social connection.

The results of this modified grounded theory analysis suggest that while motivation may be grounded in internal concern for "the body," the self is socially and institutionally embedded. This supports existing theories such as the Physical Activity Maintenance (PAM) theory, which posits that contextual variables, including "a supportive social surrounding and physical environment conductive to PA," can increase individual motivation [45]. Yet, PAM diverges from the results of this study in its assumption that Life Stress can "decrease motivation to engage in an active lifestyle." Yet in this study, OACS exercise motivations cannot be de-contextualized from uncertainties around survivorship and recurrence. In fact, the psychological impacts following major life stressor-including heightened anxiety about illness recurrence and navigating an uncertain future-serve as a powerful reason for engaging in PA. While this study did not specifically examine maintenance, there is room for future work to examine how these concepts interact with the constructs of PAM over time.

The concepts identified in this study can inform the development of future interventions to improve PA maintenance among OACS. As the results indicate, interventions that make explicit connections between exercise and cancer 
recovery, facilitate interpersonal interaction, and promote a sense of safety may be the most effective. Further, these findings can be used to help evaluate existing programs, helping determine why a specific behavior change intervention was successful or not among the OACS population, or identifying specific areas for improvement [16]. Future longitudinal studies will further clarify the interrelationship among the internal and external themes and identify which are the most salient in supporting long-term behavior change among OACS with and without a history of exercise. As this study suggests, those with a history of exercise may be more motivated by internal factors ("moving forward" from cancer through movement) while those with limited exercise experience may be more motivated by external factors (i.e., a safe and nonjudgmental clinical context where they feel comfortable testing new limits).

\section{Public Health Implications}

The social connection formed from fitness class participation was a strong motivation in this population. This aligns with the results of previous work with OACS, where social support was found to be a facilitator for informal PA uptake [27]. Therefore, the expansion of OACS-focused programming should leverage existing community groups and networks. As this study found, exercising with a consistent group of peers helps establish a routine and sense of accountability, which can be a catalyst for other sustained exercise behaviors. In addition, the connections formed through the fitness class carried into other aspects of the participants' social lives, indicating that group-based PA may help address the growing problem of older adult social isolation.

The results of this study also indicate that program context is critical to sustainability: in the present study, the medical-adjacent setting and credentials of the coach helped drive program initiation and comfort. This aligns with recent international systematic reviews-covering studies from the USA, Australia, New Zealand, Taiwan, Canada, Norway, Ireland, the Netherlands, and Puerto Rico- which found that cancer survivors have a preference for PA counseling delivered by a fitness expert from a cancer center [46, 47]. Therefore, engaging community health centers or medical staff in OACS PA program planning, especially in community-based settings, may support program acceptance and motivate program enrollment. An international systematic review also indicated preferences for home-based PA [46]. In response to the COVID-19 pandemic, the senior author (JJM) rapidly scaled a home-based integrative medicine program, which included synchronous fitness classes delivered via videoconference [48]. A recent qualitative evaluation of this study indicated that the virtual fitness classes maintained many of the aspects of social camaraderie and psychological coping as the in-person classes [49], presenting a possible accessible avenue for group-based PA.

\section{Clinical Implications}

Results from this study reveal that perceived safety—including environment and delivery — of a fitness program is a key driver of PA motivation among the OACS population. Participants also emphasized the clinical benefit of exercise. This contradicts previous work among OACS populations, where participants did not hold strong beliefs that exercise would decrease long-term treatment effects [50]. This was among a sample of adults who had not yet begun an exercise regimen, speaking to the fact that clinical communication about the benefit of exercise both before and throughout a PA program can encourage uptake and maintenance. However, the present study provides evidence that there is a lack of communication between OACS and their care team around exercise, which has been corroborated in previous studies among older adult populations [11, 51]. Thus, training clinicians to effectively communicate about PA to OACS is critical to reinforcing safety and appropriateness of post-cancer exercise. As the results indicate, the clinical value that participants in this study placed on exercise speaks to the value of cultivating a trusting relationship between patients and the medical institution during treatment, which can be carried forward into conversations about survivorship.

Concerns about safety and efficacy delay PA initiation. Therefore, building trust in the clinical encounter from the outset, including assessing a patient's PA habits and goals, may be key to the initiation of safe PA posttreatment. Grounding clinical conversations around OACS motivations for exercise uncovered in this study—such as reducing risk of recurrence and promoting social engagement-may enhance the effectiveness of physician communication. Many participants in the study also framed PA as a key component of healthy survivorship, preferring to think of it as a "prescription" to stay motivated. This framing may be a useful device for clinicians encouraging their patients to exercise. At a policy level, this provides an early indication that new PA prescribing initiatives-like those in Australia [52] - may have a positive impact on OACS motivation and behavior.

\section{Limitations}

Limitations of this study include a homogenous study sample that was only English-speaking. These demographics reflect the composition of hospital catchment area, particularly those who can regularly attend in-person classes. There may be some response bias within the high self-rated global physical health scores since many surveys were completed post-exercise, fostering an enhanced sense of strength and resiliency; increased social integration and reduced isolation 
compared to peers of a similar age may account for relatively high global mental health scores. However, these scores were comparable to a large, cross-sectional sample of older adult cancer survivors with similar demographics (mean age 72,60\% female, 90\% white), as well as a sample of US cancer survivors of all ages [53, 54]. Finally, the majority female sample is attributed partly to sampling a breast cancer-specific fitness class, and fits well with previous literature that the older women comprise the majority of older adult PA programs [29]. There may be selection bias in that only participants who had a strongly positive or negative experience would agree to participate in an interview and share feedback. As these data were initially collected as part of a program evaluation, we did not collect information from the medical record on treatment received, disease stage, other diagnoses, BMI, or demographic information such as education or income.

We did not collect quantitative data on overall activity level, which would have allowed us to examine the extent to which participation in the fitness classes contributed to overall lifestyle changes. We were also unable to interview those who had discontinued participation in the classes, as well as physically inactive cancer survivors. Therefore, we were not able to identify potential reasons for inactivity. In specifically aiming to understand the motivations of OACS who participate in fitness classes, we did not obtain the perspectives of those who prefer to exercise independently. For these individuals, the contextual, externally driven motivations identified in this study - such as the camaraderie that comes with exercising among a community of survivors-may be less salient. However, while the goal of the study was to examine the rationale for participation in the fitness classes, some of the findings may usefully serve as a starting point to further investigate motivators of uptake of PA after cancer treatment, as well as maintenance. Therefore, our findings, while not directly relevant to those not exercising or individually exercising per se, provide useful evidence for future research to identify factors that may motivate inactive survivors.

In addition, the non-diverse sample, and the fact that all participants were recruited from a single large academic medical center, limits the generalizability of the study. While over $50 \%$ of comprehensive cancer centers in the USA now offer fitness classes for patients and their loved ones [34], many survivors are treated in community settings. However, many of the motivations identified here may also be present among the no-cost, community-based PA programs, such as the YMCA Livestrong program [35], which can be evaluated in future studies.

\section{Future Directions}

This was a modified grounded theory study to generate insights on specific PA rationales among OACS who are voluntarily active in fitness classes at a cancer center. Future study should evaluate the extent to which the concepts identified in this study apply to OACS attendees communitybased fitness classes. Future longitudinal studies will also identify the extent to which the internal and external motivations identified in this study serve as potent motivators that sustain long-term behavior change and PA maintenance. Finally, the key findings of this study highlight the importance of social structure and setting for impactful OACS fitness classes. Future work should test various clinical delivery models-including such as the in-person, virtual, and community partnerships- to reduce the barriers for engaging in fitness among older survivors, particularly for those from underserved populations.

\section{Conclusion}

Psycho-oncologists have stated that "hope is the act of creating an uncertain future" [55]. Through engaging in weekly fitness classes, the participants in this study perceive themselves to be taking an active role in shaping their uncertain futures through strengthening their physical body and social connections. In doing so, PA becomes an act of building hope during recovery. The nuanced dimensions of OACS motivations have presented a useful model of ways to move forward after a fundamental change: exercise is a way for older adults to tap into internal and external motivators that support cancer survivorship and maintenance. As motivations for sustained PA center on supporting long-term cancer recovery, PA can attend to the psychosocial, as well as physiological, needs of this population. Through the motivations identified in this study, PA programming can be created and tailored to serve the needs of the OACS population.

\section{Appendix I. Interview Guide}

\section{Introduction to Program}

1. Let's begin by thinking back to your first fitness class. Can you tell me about what that experience was like?

a. What concerns did you have before starting?

2. How did you first become interested in the fitness classes?

a. How did you learn about the program?

b. How long have you been attending classes?

3. Did anyone on your care team (doctor, nurse, oncologist) talk to you about exercise? When did this conversation happen? 
a. What were their recommendations?

\section{Motivators and Barriers}

4. How would you describe your overall experience participating in the classes so far?

a. What do you see as the most positive aspects of the program?

b. What would you like to see improved?

5. How often do you attend classes?

6. What are some of the reasons that you look forward to attending, week by week?

7. Is there anything that makes it challenging to come to classes each week?

\section{Perception of Benefit}

8. What were your exercise habits like before you began attending the fitness classes?

a. How did this influence your decision to participate in the program?

b. How have they changed since you began the classes?

9. Since you've began the classes, have you noticed any changes in how you've felt? a. Are there any activities that you are now able to do, that you weren't able to do before you began participating?

\section{Program Feedback}

10. If you could describe the class to other patients, what would you say?

a. What would motivate you to refer another patient to this program?

b. Who do you think would benefit from this fitness program?)

11. Is there anything that you think is missing from the classes?

\section{Appendix 2: Modified Grounded Theory Analysis Protocol}

Transcripts were coded in three phases. Transcripts were first reviewed in their entirety using an open coding process, wherein significant statements were highlighted and assigned a descriptive or interpretive label. From this review, an initial codebook was developed incorporating constructs of self-determination theory and social cognitive theory-two dominant theories of exercise motivation [1, 2, and 3]- as well novel constructs that inductively emerged from the data. Through a process of axial coding, the codebook was solidified and applied across all transcripts, so that significant categories may be identified and grouped under thematic labels supported by the text, thereby grounding each category in the data. The primary coder held weekly consensus meetings with a member of the study team with expertise in qualitative research (AM), who reviewed the emergent categories and offered suggestions for refinement. The final phase consisted of a selective coding process, wherein significant statements grouped within the categories of interest were re-coded to identify primary themes and build a framework. Coded categories were revisited by KAL and AM to identify instances of thematic saturation. Consistent with the modified grounded theory process, this method of iterative comparison and review was used to resolve discrepancies within the codebook, enhance intra-rater reliability, and identify emergent categories to inform a framework.

\section{References}

1. Pudkasam, $\mathrm{S}$ et al. Physical activity and breast cancer survivors: Importance of adherence, motivational interviewing and psychological health. Maturitas, 2018;116: 66-72. https://doi.org/10.1016/j.maturitas.2018.07.010

2. Stacey, F.G., James, E.L., Chapman, K. et al. A systematic review and meta-analysis of social cognitive theory-based physical activity and/or nutrition behavior change interventions for cancer survivors. J Cancer Surviv 9, 305-338 (2015). https://doi.org/10.1007/s11764-014-0413-z

3. B.M. Pinto, J.T. Ciccolo, Physical activity motivation and cancer survivorship, Physical Activity and Cancer, Springer, 2010, pp. 367-387.

Acknowledgements The authors would like to thank Dr. Nina Parikh, $\mathrm{PhD}$, for her guidance and support throughout this study.

Funding Research reported in this publication was supported by a National Institutes of Health Support Cancer Center Support Grant (P30-CA008748-53) which partially supports the Patient-Reported Outcomes, Community-Engagement, and Language Core Facility used in carrying out this investigation.

\section{Declarations}

Ethical Approval This analysis was conducted retrospectively from data obtained for program evaluation purposes. This study involving human participants was in accordance with the ethical standards of the institutional and national research committee and with the 1964 Helsinki Declaration and its later amendments or comparable ethical standards. The Institutional Review Board of Memorial Sloan Kettering Cancer Center approved this study.

Informed Consent Informed consent was obtained from all individual participants included in the study. 
Competing of Interest The authors declare no competing interests.

\section{References}

1. Bluethmann SM, Mariotto AB, Rowland JH. Anticipating the "Silver Tsunami": prevalence trajectories and comorbidity burden among older cancer survivors in the United States. Cancer Epidemiol Biomarkers Prev. 2016;25(7):1029-36.

2. American Cancer Society, Cancer Facts \& Figures 2020.

3. Chlebowski RT. Nutrition and PA influence on breast cancer incidence and outcome. Breast. 2013;22(Suppl 2):S30-7.

4. Smith TM, Broomhall CN, Crecelius AR. Physical and psychological effects of a 12-session cancer rehabilitation exercise program. Clin J Oncol Nurs. 2016;1;20(6):653-659.

5. Courneya KS, Mackey JR, Bell GJ, Jones LW, Field CJ, Fairey AS. Randomized controlled trial of exercise training in postmenopausal breast cancer survivors: cardiopulmonary and quality of life outcomes. J Clin Onc. 2003;21(9):1660-8.

6. Paxton RJ, et al. Associations among PA, body mass index, and health-related quality of life by race/ethnicity in a diverse sample of breast cancer survivors. Cancer. 2012;118(16):4024-31.

7. Naraphong W, et al. Exercise intervention for fatigue-related symptoms in Thai women with breast cancer: a pilot study. Nurs Health Sci. 2015;17(1):33-41.

8. Demark-Wahnefried, W., Rogers, L. Q., Gibson, J. T., et al. Randomized trial of weight loss in primary breast cancer: impact on body composition, circulating biomarkers and tumor characteristics. Int. J. Cancer 2019

9. Lee K, Tripathy D, Demark-Wahnefried W, et al. Effect of aerobic and resistance exercise intervention on cardiovascular disease risk in women with early-stage breast cancer: a randomized clinical trial. JAMA Oncol. 2019;5(5):710-4.

10. Daum CW, Cochrane SK, Fitzgerald JD, et al. Exercise interventions for preserving physical function among cancer survivors in middle to late life. J Frailty Aging. 2016;5(4):214-24.

11. Kenzik K, Pisu M, Fouad MN, Martin MY. Are long-term cancer survivors and physicians discussing health promotion and healthy behaviors? J Cancer Surviv. 2016;10(2):271-9.

12. Guerard EJ, Nightingale G, Bellizzi K, Burhenn P, et al. Survivorship care for older adults with cancer: U13 conference report. J Geriatr Oncol. 2016;7(4):305-12.

13. Grimmett $\mathrm{C}$, Corbett $\mathrm{T}$, Brunet J, et al. Systematic review and meta-analysis of maintenance of PA behaviour change in cancer survivors. Int J Behav Nutr Phys Act. 2019;16(1):37.

14. Harper K., Schmidt F.W., Beacham J.E., et L. The role of social cognitive processing theory and optimism in positive psychosocial and physical behavior change after cancer diagnosis and treatment. Psycho-Oncology. 2007; 16: 79-91

15. Faro, J.M., Mattocks, K.M., Mourao, D. et al. Experiences and perceptions of referrals to a community-based physical activity program for cancer survivors: a qualitative exploration. BMC Health Serv Res. 2021;21(358).

16. Courneya K.S., Karvinen K.H., Vallance J.K. Exercise motivation and behavior change. In: Feuerstein M. (eds) Handbook of cancer survivorship. Springer, Boston, MA, 2007.

17. Mazzoni AS, Carlsson M, Berntsen S, et al. "Finding my own motivation"- - a mixed methods study of exercise and behaviour change support during oncological treatment. Int J Behav Med. 2019;26:499.

18. Midtgaard J, Hammer NM, Andersen C, Larsen A, Bruun DM, Jarden M. Cancer survivors' experience of exercise-based cancer rehabilitation-a meta-synthesis of qualitative research. Acta Oncol. 2015;54(5):609-17.
19. Devereux-Fitzgerald A, Powell R, Dewhurst A, French DP. The acceptability of PA interventions to older adults: a systematic review and meta-synthesis. Soc Sci Med. 2016;158:14-23.

20. Morgan GS, Willmott M, Ben-Shlomo Y, Haase AM, Campbell RM. A life fulfilled: positively influencing PA in older adults-a systematic review and meta-ethnography. BMC Public Health. 2019;19(1):362.

21. Miller K, Merry B, Miller J. Seasons of survivorship revisited. J Cancer. 2008;14(6):369-74.

22. Hewitt M, Greenfield S, Stovall E. From cancer patient to cancer survivor: lost in transition. Washington, DC: The National Academic Press; 2006.

23. Jefford M, Karahalios E, Pollard A, et al. Survivorship issues following treatment completion: results from focus groups with Australian cancer survivors and health professionals. J Cancer Surviv. 2008;2:20-32.

24. Deimling GT, Bowman KF, Sterns S, Wagner LJ, Kahana B. Cancer-related health worries and psychological distress among older adult, long-term cancer survivors. Psychooncology. 2006;15:306-20.

25. Pieters HC, Heilemann MV. "Once you're 82 going on 83, surviving has a different meaning": older breast cancer survivors reflect on cancer survivorship. Cancer Nurs. 2011;34(2):124-33.

26. Pieters H. "I' $m$ still here": resilience among older survivors of breast cancer. Cancer Nurs. 2016;39(1):E20-8.

27. Whitehead S, Lavelle K. Older breast cancer survivors' views and preferences for PA. Qual Health Res. 2009;19(7):894-906. https://doi.org/10.1177/1049732309337523.

28. Costello E, Kafchinski M, Vrazel J, Sullivan P. Motivators, barriers, and beliefs regarding PA in an older adult population. J Geriatr Phys Ther. 2011;34(3):138-47.

29. Cheville AL, Dose AM, Basford JR, Rhudy LM. Insights into the reluctance of patients with late-stage cancer to adopt exercise as a means to reduce their symptoms and improve their function. J Pain Symptom Manage. 2012;44(1):84-94.

30. Owusu C, Antognoli E, Nock N, et al. Perspective of older AfricanAmerican and Non-Hispanic white breast cancer survivors from diverse socioeconomic backgrounds toward PA: A qualitative study. J Geriatr Oncol. 2018;9(3):235-42.

31. Hardcastle SJ, Galliott M, Lynch BM, et al. 'If I Had Someone Looking Over My Shoulder...': exploration of advice received and factors influencing PA among non-metropolitan cancer survivors. Int J Behav Med. 2019;26(5):551-61.

32. Mikkelsen MK, Nielsen DL, Vinther A, Lund CM, Jarden M. Attitudes towards PA and exercise in older patients with advanced cancer during oncological treatment - A qualitative interview study. Eur J Oncol Nurs. 2019;41:16-23.

33. Perry CK, Ali WB, Solanki E, Winters-Stone K. Attitudes and beliefs of older female breast cancer survivors and providers about exercise in cancer care. Oncol Nurs Forum. 2020;47(1):56-69.

34. Yun H, Sun L, Mao JJ. Growth of integrative medicine at leading cancer centers between 2009 and 2016: a systematic analysis of NCI-designated comprehensive cancer center websites, JNCI Monographs, 2017; 2017(52): lgx004

35. Heston AH, Schwartz AL, Justice-Gardiner H, Hohman KH. Addressing physical activity needs of survivors by developing a community-based exercise program: LIVESTRONG(R) at the YMCA. Clin J Oncol Nurs. 2015;19(2):213-7.

36. Faro, J.M., Mattocks, K.M., Mourao, D. et al. Experiences and perceptions of referrals to a community-based physical activity program for cancer survivors: a qualitative exploration. BMC Health Serv Res. 2021;21(358).

37. Guest G, Bunce A, Johnson L. How many interviews are enough? An experiment with data saturation and variability. Field Methods. 2006;18:59-82. 
38. Hays RD, Bjorner J, Revicki RA, Spritzer KL, Cella D. Development of physical and mental health summary scores from the Patient Reported Outcomes Measurement Information System (PROMIS) global items. Qual Life Res. 2009;18(7):873-80.

39. Creswell J, Poth C, 4ed. Qualitative inquiry and research design: choosing among five approaches. Thousand Oaks, California: Sage Publications, 2018.

40. StataCorp. Stata Statistical Software: Release 15. College Station, TX: StataCorp LLC. 2017.

41. Charmaz K. Constructing grounded theory: a practical guide through qualitative analysis. London: Sage Publications; 2006.

42. Hallberg LRM. The "core category" of grounded theory: making constant comparisons. Int J Qual Stud Health Well-being. 2006;1(3):141-8.

43. Bazeley P, Jackson K. Qualitative data analysis with NVivo. London: Sage Publications; 2013.

44. Phoenix C, Bell SL. Beyond "Move More": feeling the rhythms of PA in mid and later-life. Soc Sci Med. 2019;231:47-54.

45. Nigg CR, Borrelli B, Maddock J, Dishman RK. A theory of physical activity maintenance. Appl Psychol. 2008;57:544-60.

46. Wong JN, McAuley E, Trinh L. Physical activity programming and counseling preferences among cancer survivors: a systematic review. Int J Behav Nutr Phys Act. 2018;15(1):48.

47. Buffart LM, Galvão DA, Brug J, Chinapaw MJ, Newton RU. Evidence-based physical activity guidelines for cancer survivors: current guidelines, knowledge gaps and future research directions. Cancer Treat Rev. 2014;40(2):327-40.

48. Trevino KM, Raghunathan N, Latte-Naor S, et al. Rapid deployment of virtual mind-body interventions during the COVID-19 outbreak: feasibility, acceptability, and implications for future care. Support Care Cancer. 2021;29:543-6.
49. Emard N, Lynch KA, Liou KT, et al. Virtual mind-body programming for patients with cancer during the COVID-19 pandemic: qualitative study. JMIR Cancer 2021;7(2):e27384.

50. Hirschey R, Docherty S, Pan W, Lipkus I. Exploration of exercise outcome expectations among breast cancer survivors. Cancer Nurs. 2017;40(2):E39-46.

51. Siembida EJ, Kent EE, Bellizzi KM, Wilder SA. Healthcare providers' discussions of PA with older survivors of cancer: potential missed opportunities for health promotion. J Geriatr Oncol. 2020;11(3):437-43.

52. Hayes SC, Newton RU, Spence RR, Galvão DA. The Exercise and Sports Science Australia position statement: exercise medicine in cancer management. J Sci Med Sport. 2019;22(11):1175-99.

53. Pergolotti M, Deal AM, Williams GR, et al. Activities, function, and health-related quality of life (HRQOL) of older adults with cancer. J Geriatr Onc. 2017;8(4):249-54.

54. Weaver KE, Forsythe LP, Reeve BB, et al. Mental and physical health-related quality of life among U.S. cancer survivors: population estimates from the 2010 National Health Interview Survey. Cancer Epidemiol Biomarkers Prev. 2012 Nov;21(11):2108-17.

55. Breitbart, W. Meaning-centered psychotherapy in the cancer setting: finding meaning and hope in the face of suffering. Oxford University Press, 2016.

Publisher's Note Springer Nature remains neutral with regard to jurisdictional claims in published maps and institutional affiliations. 